ANATOLIA Anatolia Antiqua

ANTIQUA

Revue internationale d'archéologie anatolienne

XXVII 2019

Varia

\title{
Aksel et la viole de gambe
}

Christine Kepinski

\section{(2) OpenEdition}

Journals

Édition électronique

URL : https://journals.openedition.org/anatoliaantiqua/1231

DOI : 10.4000/anatoliaantiqua. 1231

Éditeur

IFEA

Édition imprimée

Date de publication : 31 décembre 2019

Pagination : 13-16

ISBN : 978-2-36245-080-8

ISSN : 1018-1946

\section{Référence électronique}

Christine Kepinski, « Aksel et la viole de gambe », Anatolia Antiqua [En ligne], XXVII | 2019, mis en ligne le 26 février 2020, consulté le 16 mars 2022. URL : http://journals.openedition.org/anatoliaantiqua/ 1231 ; DOI : https://doi.org/10.4000/anatoliaantiqua.1231 


\title{
ANATOLIA ANTIQUA ESKI ANADOLU
}

\author{
XXVII
}

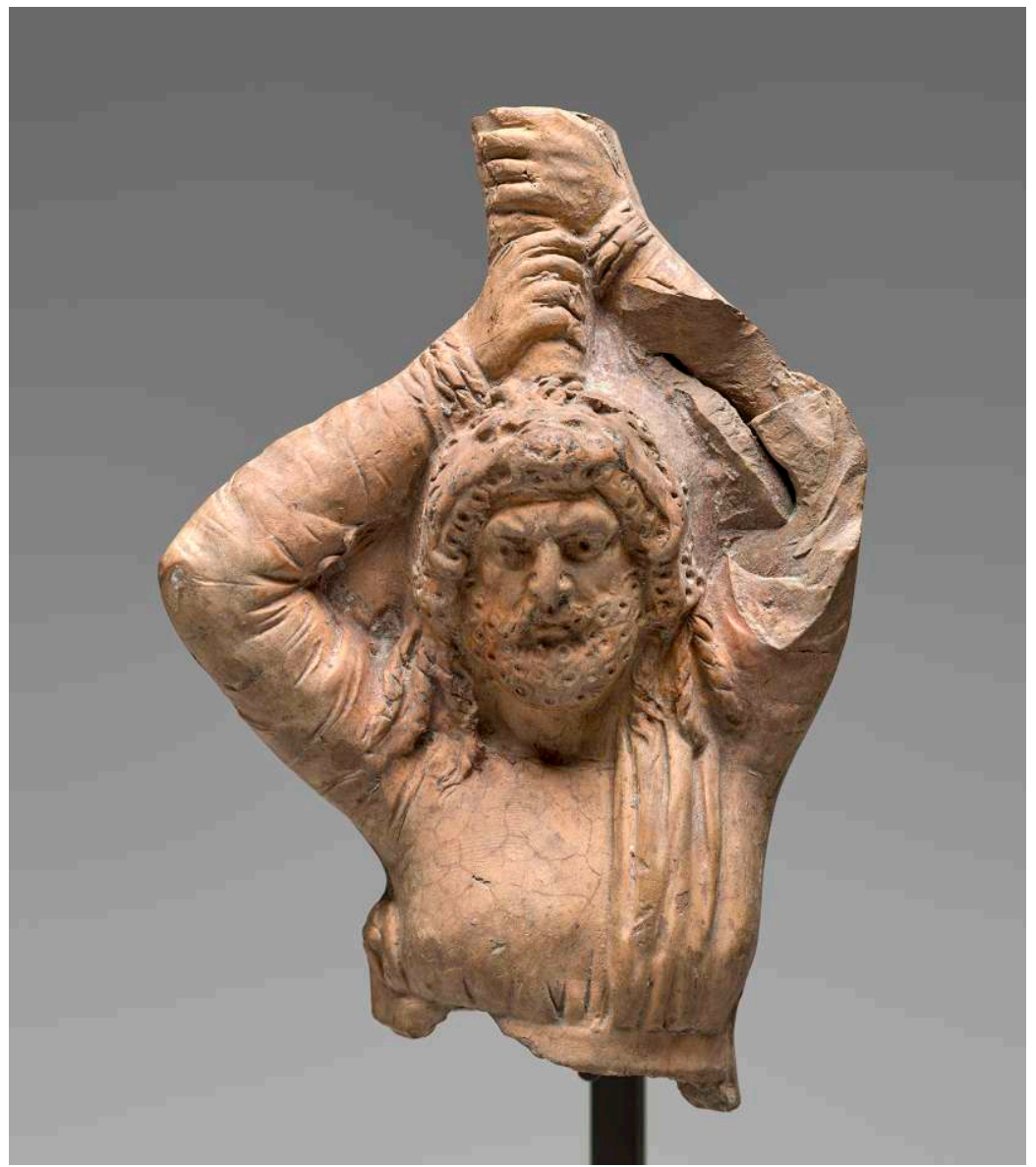

INSTITUT FRANÇAIS D'ETUDES ANATOLIENNES GEORGES-DUMEZIL CNRS USR 3131

DE BOCCARD 


\section{ANATOLIA ANTIQUA ESKI ANADOLU XXVII}

Recueil de travaux publiés par

l'Institut Français d'Etudes Anatoliennes Georges-Dumézil Istanbul

édite par

Deniz GENCEOLU et Martin GODON

\section{OFFPRINT / AYRIBASIM}

INSTITUT FRANÇAIS D'ETUDES ANATOLIENNES GEORGES - DUMEZIL CNRS USR 3131

DE BOCCARD Edition - Diffusion

11, rue de Médicis

75006 Paris 


\section{Comité de lecture}

Mme Catherine ABADIE-REYNAL, Université Lumière-Lyon 2.

Mme Nur BALKAN-ATLI, Université d'Istanbul.

M. Dominique BEYER, Université de Strasbourg.

M. Wolfgang BLÜMEL, Université de Cologne.

Mme Isabella CANEVA, Université de Salento (Lecce). Mme Marie-Claire CAUVIN, CNRS.

M. Jacques des COURTILS, Université Michel de Montaigne Bordeaux 3.

M. Alain DAVESNE, Université d'Orléans.

Mme Roberta FABIANI, Université de Pérouse. Mme Véronique FRANÇOIS, CNRS.

Mme Marcella FRANGIPANE, Université de Rome, "La Sapienza". Mme Marie-Henriette GATES, Université Bilkent (Ankara).

M. Harald HAUPTMANN, Université de Heidelberg.

M. Wilfried HELD, Université de Marburg.

M. Jean-Louis HUOT, Université Paris 1 Panthéon-Sorbonne.

M. Francis JOANNES, Université Paris 8.

Mme Christine KEPINSKI, CNRS.

M. Koray KONUK, CNRS.

Mme Catherine KUZUCUOĞLU, CNRS.

M. René LEBRUN, Université catholique de Louvain.

M. Christian LEROY, Université Paris 1 Panthéon-Sorbonne.

Mme Vasilica LUNGU, Institute of South-Eastern European Studies, Académie Roumaine.

Mme Raffaella PIEROBON-BENOIT, Université de Naples.

Mme Catherine MARRO, CNRS.

M. Philipp NIEWÖHNER, Université d'Oxford.

M. Alistair NORTHEDGE, Université Paris IV-Sorbonne.

Mme Mihriban ÖZBAŞARAN, Université d'Istanbul.

Mme Christine ÖZGAN, Université Mimar Sinan des Beaux-Arts (Istanbul).

M. Francis PROST, Ecole Normale Supérieure (Paris).

M. Cemal PULAK, Université de Texas A\&M.

M. Jean-Pierre SODINI, Académie des Inscriptions et Belles-Lettres (Paris).

M. Rahmi Hüseyin ÜNAL, Université d'Ege (İzmir).

Ce volume a été composé par les soins

de l'Institut Français d'Etudes Anatoliennes-Georges Dumézil

et Zero Prod. Ltd. Abdullah Sok. 17 Taksim 34433 Beyoğlu, Istanbul, Turquie, et imprimé par Matsis Matbaa Hizmetleri San. Ve Tic. Ltd. Şti.

La publication a pu en être réalisée grâce au concours financier

du Ministère de l'Europe et des Affaires étrangères, et du Centre National de la Recherche Scientifique.

CC 2019, Institut Français d'Etudes Anatoliennes Georges-Dumézil - Istanbul

La loi du 11 mars 1957 n'autorisant, aux termes des alinéas 2 et 3 de l'article 41, d'une part, que les "copies ou reproductions strictement réservées à l'usage privé du copiste et non destinées à une utilisation collective" et, d'autre part, que les analyses et les courtes citations dans un but d'exemple et d'illustration, "toute" représentation ou reproduction intégrale, ou partielle, faite sans le consentement de l'auteur ou de ses ayants droit ou ayants cause, est illicite (alinéa 1er de l'article 40).

Cette représentation ou reproduction, par quelque procédé que ce soit, constituerait donc une contefaçon sanctionnée par les articles 425 et suivants du Code Pénal. 


\section{TABLE DES MATIERES}

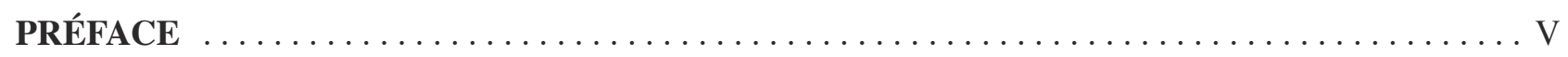

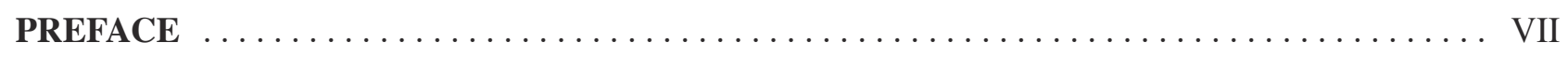

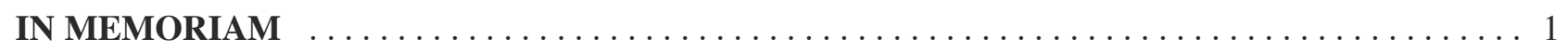

Burçin ERDOĞU et Özlem ÇEVIK

A Comparative Study of the Sixth Millennium BC Marble Bracelets from Ulucak and Uğurlu . . . . . 29

Martin GODON et Ozan ÖZBUDAK

Early Chalcolithic Pottery from Tepecik-Çiftlik and Gelveri, Central Anatolia:

From Local to Supra-Regional Ceramic Style $\ldots \ldots \ldots \ldots \ldots \ldots \ldots \ldots \ldots \ldots \ldots \ldots \ldots \ldots \ldots \ldots \ldots \ldots \ldots$

Vladimir SHELESTIN

On the Origin of Küçükçekmece Iron Figurines $\ldots \ldots \ldots \ldots \ldots \ldots \ldots \ldots \ldots \ldots \ldots \ldots \ldots \ldots \ldots \ldots \ldots \ldots \ldots$

Akın ERSOY et Ludovic LAUGIER

Sculptures grecques et romaines de Smyrne, découvertes récentes $\ldots \ldots \ldots \ldots \ldots \ldots \ldots \ldots \ldots 7$

Élisabeth GOUSSARD, Raphaëlle CHEVALLIER, Olivier HENRY et Christophe BOST

Labraunda 2018, Étude d'un poids décoré et d'une couronne :

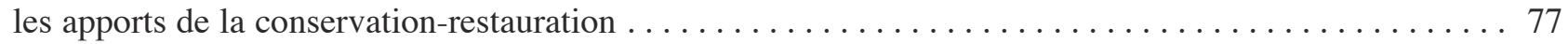

Isabelle HASSELIN ROUS

Les figurines en terre cuite de Tarse en Cilicie à l'époque romaine impériale $\ldots \ldots \ldots \ldots \ldots \ldots$

İnci TÜRKOĞLU

Early Byzantine Structure at Gerenkuyu Mevkii of Yal1-Bodrum . . . . . . . . . . . . . . . . . 109

Anca DAN, Sait BAŞARAN, Helmut BRÜCKNER, Ercan ERKUL, Anna PINT,

Wolfgang RABBEL, Lyudmila SHUMILOVSKIKH, Dennis WILKEN and Tina WUNDERLICH

Ainos in Thrace: Research Perspectives in Historical Geography and Geoarchaeology . . . . . . . 127

Hülya ATAŞCIOĞLU AYKUL, M. Baha TANMAN, Miguel Ángel ESCOBAR-CLARÓS

A Note on the Turkish Lot III / 1891 from the Bab el-Gasus Cache (Egypt), kept at the Istanbul

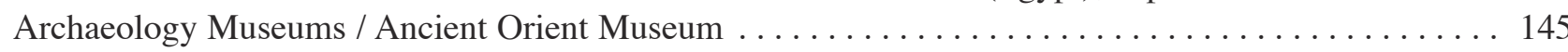

\section{CHRONIQUES DES TRAVAUX ARCHEOLOGIQUES EN TURQUIE 2018}

Kenan IŞIK, Bülent GENÇ, Vedat TIMMUR et Rıfat KUVANÇ

Two Column Bases From Mawan in the Hakkâri Province (Southeast Corner of Turkey):

A New Achaemenid Center? 


\section{Çiğdem MANER}

Preliminary Report on the Sixth Season of the Konya Ereğli, Karapınar, Halkapınar and

Emirgazi Survey Project (KEYAR) 2018 .

Erkan KONYAR, Bülent GENÇ, Can AVCI et Armağan TAN

Excavations at the Old City, Fortress, and Mound of Van: Work in 2018

Olivier HENRY et J. BLID, Chr. BOST, N. CARLESS-UNWIN, R. CHEVALLIER, G. ÇIMEN,

A. EYIGÖR, A. FREJMAN, E. GOUSSARD, V. LUNGU, A. SITZ, A. MUSAT-STREINU,

B. VERGNAUD

Labraunda 2018

M. SEYER, A. DOLEA, P. M. BES, D. Zs. SCHWARCZ, S. BAYBO, A. K. L. LEUNG,

U. QUATEMBER, M. WÖRRLE, H. BRÜCKNER, F. STOCK, A. SYMANCZYK, G. STANZL,

K. KUGLER, B. YENER-MARKSTEINER

The Excavation at Limyra/Lycia 2018: Preliminary Report

Alessandra RICCI

The Küçükyalı Arkeopark (Istanbul), 2016-2018: Excavation, Conservation, Cultural Heritage and Public Archaeology

Nergis GÜNSENIN

A Günsenin IV Amphora from Küçükyalı 


\section{Christine KEPINSKI*}

\section{AKSEL ET LA VIOLE DE GAMBE}

La disparition d'Aksel nous bouleverse. J'aimerais ici lui donner la parole pour illustrer la richesse de son tempérament. Dans des lettres généralement destinées à accompagner des traductions de textes divers, des informations, des conseils, Aksel aimait aussi échanger sur sa passion pour la musique.

Extraits de courrier reçu par fax :

02/08/1995

PS bis (qui n'a rien à voir avec ce qui précède) : Depuis quelque temps, j'ai une passion pour la viole de gambe. Cette passion est tellement forte que j'ai décidé d'essayer d'apprendre à en jouer. J'ai déjà trouvé un prof qui veut bien m'apprendre (il est celliste mais se débrouille aussi à la viole). Je viens de me rappeler que tu joues de la viole et que tu fais partie des pionniers de la résurrection de cet instrument. Je suis à la recherche d'un instrument. Il est bien sûr, impossible d'en trouver en Turquie. Je pense donc l'acheter en France. Quel pourrait être le prix d'une viole de gambe ? 10 000, $20000 \mathrm{~F}$ ou plus ? Existe-t-il des violes modernes (peutêtre moins chères ?) ou tous les instruments sont-ils d'époque. Y-a-t-il des méthodes pour apprendre ? Je serais très content si tu pouvais me renseigner sur ces points. Merci d'avance.

\section{3/08/1995}

En ce qui concerne la viole de gambe, je ne sais pas comment te remercier. Sans toi je n'aurais jamais pu avoir des indications si claires et si détaillées. La précision de l'information m'a beaucoup aidé à prendre une décision rapide. Je penche donc pour la viole de $15.000 \mathrm{~F}$ (+ étui + archet + cordes + méthode). Je ne sais pas ce qu'il faut faire pour passer la commande. Est-il possible que tu contactes le luthier en question pour lui demander les conditions (le prix exact, le montant des arrhes, le délai de fabrication). Peut-être fera-t-il une sorte de devis. Une fois ces conditions établies, je t'enverrai la somme correspondante aux arrhes demandées pour que l'on puisse passer la commande.

\section{$12 / 02 / 1996$}

En ce qui concerne la musique, j'ai pas mal travaillé sur la clef d'ut qui ne me semble pas poser de grandes difficultés de lecture, car le décalage par rapport à la clef de sol est d'un demi ton (à l'octave du bas bien sûr). D'autre part je me suis procuré une vingtaine de CD de viole (les 5 livres de Marais, les 2 livres de SainteColombe, ainsi que Couperin, Caix d'Hervelois, Forqueray, de la musique anglaise de consorts de violes etc.), interprétés pour la plupart par Jordi Savall et Wieland Kuijken. En les écoutant, j’essaye de me familiariser avec cette musique. C'est tout ce que je peux faire pour le moment. Une fois que j'aurai l'instrument et la méthode, j'essayerai d'abord de travailler tout seul. Mais si ça ne marche pas comme ça, je demanderai l'aide d'un violoncelliste, au moins pour certains détails. J'attends d'autre part tes instructions pour te faire parvenir la somme nécessaire pour payer l'instrument, l'archet, la méthode, le boitier, les cordes etc. Je pense que je pourrais te l'envoyer courant avril. Pour ce qui concerne du transport, es-tu sûre que ça ne va pas t'encombrer pendant le voyage? On pourrait peut-être trouver une autre solution qui te dérange moins.

*) Christine Kepinski, Directrice de recherche Emérite Maison René-Ginouvès ArScAn Archéologies et Sciences de l'Antiquité 21, allée de l’Université 92023- Nanterre Cedex christine.kepinski@gmail.com 
$10 / 07 / 1996$

Je suis vraiment désolé d'avoir confisqué à Istanbul tes cordes de rechange. J'espère que tu pourras en trouver près de chez toi ou de l'Institut d'Art, sans aller à l'autre bout de Paris.

Comme je te l'avais dit dans notre entrevue rapide à Istanbul, je m'exerce régulièrement à la viole à partir de la méthode anglaise et ça à l'air de ne pas aller trop mal. J'arrive à produire un son satisfaisant et commence à avoir une certaine agilité (tout à fait relative) des doigts.

L'autre jour j'ai acheté un CD d'Alfred Deller qui chante du Purcell accompagné d'une basse de viole. Pour certains morceaux simples avec basso ostinato, j'ai pu transcrire la partie basse (ma dictée n'est pas mal), ce qui fait que maintenant je peux accompagner Deller avec ma viole !...

03/04/1997

Enfin je suis bien sûr intéressé par ta proposition de me prêter ton dessus de viole pour deux mois, mais d'une part, tu seras déjà chargée par les affaires de la mission (quand je pense que l'an dernier tu m'avais apporté l'énorme basse malgré tout ton chargement, je ne trouve pas de mots assez forts pour t'exprimer ma reconnaissance), et d'autre part, moi-même je serai en mission à partir du 15 juin, ce qui m'obligera à laisser l'instrument à quelqu'un de l'Institut afin que tu puisses le récupérer à ton retour. En ce qui concerne le duo, je suis très flatté mais je crains de ne pas être à la hauteur.

Ce qui pourrait être envisageable c'est que tu sois l'invitée de mon émission sur la viole de gambe. En effet je termine mon cycle de 25 émissions sur ce sujet le samedi 26 avril. Comme tu pars le 25, je pourrais m'arranger pour avoir un studio le jeudi 24 pour enregistrer l'émission d'avance (je fais d'habitude du direct). Je pense qu'il serait intéressant pour le public turc d'avoir l'opinion d'une gambiste française sur la musique de viole en général, la renaissance de cette musique en France et les difficultés qui sont liées à une certaine diminution de l'intérêt du public ces derniers temps etc. Si cela te dit tu pourrais même interpréter quelques morceaux (de toutes façons, ce ne sera pas du direct, on pourra donc écouter et réenregistrer si besoin). Fais-moi donc savoir ton opinion sur cette proposition afin que je puisse arranger les choses.

Enfin pourrais-je te demander de m'apporter 2 cordes de ré aigu et 2 cordes de la (ce sont celles qui cassent le plus) si tu as l'occasion de passer par une boutique de musique.

\section{$19 / 11 / 1997$}

Je te remercie infiniment d'avance pour la nouvelle méthode de viole de gambe dont tu m'annonces l'envoi prochain. Malheureusement les campagnes de Kastamonu et de Horum Höyük ont eu des conséquences néfastes sur mon programme d'entrainement. Presque 3 mois d'arrêt total! J'essaye de rattraper le retard. D'autre part je suis en train de devenir le promoteur $n^{\circ} 1$ en Turquie de la musique de viole et de l'instrument : en effet dans une radio locale d'Istanbul - Açik Radyo 94.9 hhz ${ }^{1}$ - j'ai commencé un cycle de 26 émissions (à raison de $1 \mathrm{~h}$ par semaine chaque samedi matin) sur ce sujet (le programme est intitulé « Tous les matins du samedi »!!!.j'ai fait la première émission sur la présentation générale de l'instrument, la $2^{\text {ème }}$ sur l'école anglaise (Christopher Tye) et cette semaine je continue avec Dowland etc. On a fait aussi des reportages sur moi, un fou qui essaye d'apprendre à jouer un instrument ancien et rare, après 40 ans ! etc.

\section{9/12/1997}

Mes activités sur le terrain qui ont duré plus de 3 mois ont été assez néfastes pour ma viole. J'essaye, depuis mon retour il y a près d'un mois et demi, d'atteindre à nouveau le niveau que j'avais avant mon départ. Je n'y suis arrivé qu'il y a quelques jours. Il a fallu retravailler presque tous les exercices qui ne me posaient auparavant aucun problème. De plus, les multiples tâches que je dois assumer (publications, rapports et chroniques de fouilles, chœur etc.) ne me laissent pas le temps voulu pour m'exercer. Malgré tout, j'essaye de toucher l'instrument régulièrement chaque jour, même si ce n'est, hélas, que pour une vingtaine de minutes (mais il arrive de temps en temps que je m'exerce une heure et demi).

1) L'hommage à Aksel Tibet par Aykut Köksal sur Açık Radyo : http://acikradyo.com.tr/podcast/210855 
Je voulais t'écrire une longue lettre de remerciement pour l'accordeur que tu m'as envoyé ; Mais le hasard a fait que ça soit par fax que je le fasse. Je te remercie donc infiniment pour l'engin ultra perfectionné que tu m'as envoyé. Il me permet de ne plus perdre mon temps à accorder l'instrument, mais d'utiliser le temps ainsi gagné à m'exercer. J'ai aussi pu repositionner tous les boyaux du manche qui avaient bougé avec le temps. Je suis donc ravi d'avoir cette petite boite magique. Ceci dit, il faut absolument que tu me dises combien cela t'a coûté afin que je puisse t'envoyer la somme correspondante. Si tu ne le fais pas - et je sais que tu penches pour cette solution - je ne pourrais plus rien te demander, ni cordes, ni partitions. J'insiste donc énergiquement pour que tu le fasses.

PS : Je crains que cette année encore, je ne pourrai pas te jouer les Folies d'Espagne et qu'il faudra te contenter de quelques pièces anglaises du $16^{\mathrm{e}} \mathrm{s}$.

Extraits de courrier reçu par mèl :

07/05/2004

En ce qui concerne la musique, malheureusement ma viole est devenue aussi un meuble ! Mais je garde l'espoir de pouvoir recommencer un jour. En mai prochain nous allons chanter la messe K. 427 pour double chœur de Mozart accompagné d'un orchestre qui vient des Pays Bas, et début juillet nous chanterons le Stabat Mater de Dvorak

\section{$26 / 06 / 2004$}

L'entretien avec Jordi et Monserrat s'est formidablement bien passé. Ce sont des gens qui ont su rester humbles malgré la gloire. Après l'entretien, on est allé casser la croute quelque part et puis on s'est promené dans Istanbul toute l'après-midi. Je t'envoie une photo de nous trois.

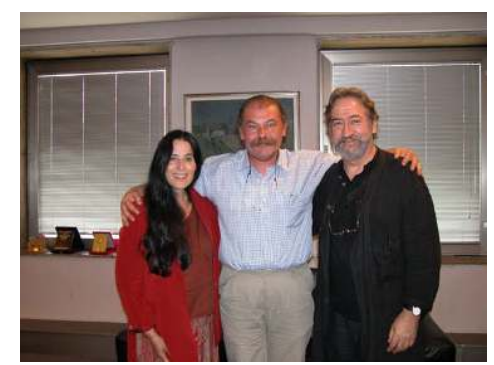

Fig. 1 : Aksel Tibet entre Montserrat Figueras et Jordi Savall !

$02 / 03 / 2011$

Quand est-ce que tu mettras les nouvelles cordes sur ta viole de gambe pour recommencer à en jouer ? N'enlève pas les anciennes cordes toutes à la fois, remplace les une à une progressivement sinon tu risques de faire bouger l'âme. Bon courage (:))

\section{0/06/2011}

Je suis à une période de stagnation concernant la viole, mon partenaire étant très occupé par des répétitions d'un chœur où il est entré nouvellement et qui lui prend tout son temps libre. D'ailleurs je partirai dans quelques semaines en fouille et il n'est pas possible que j'y emporte mon instrument. Peut-être pour les prochaines années je me procurerai une viole trebble qui sera beaucoup plus pratique à transporter.

\section{$11 / 06 / 2012$}

Par ailleurs, concernant la viole de gambe, je me suis engagé dans une affaire dépassant mes capacités, mais qui, finalement a abouti très positivement : on m'a en effet demandé de faire partie d'un petit ensemble baroque (violon, flûte traverso, clavecin, viole de gamme et chant) pour un concert-lecture à l'occasion du 
tricentenaire de Jean-Jacques Rousseau avec des morceaux de Rousseau (eh oui il a beaucoup composé), Rameau, Clérembault, Vivaldi, Pergolèse et Galuppi. A la suite de 4 mois de répétitions nous avons donné fin mai ce concert qui s'est très bien passé. On a eu beaucoup de succès. Je t'envoie en pièce jointe l'affichette du concert pour que tu voies de quoi il s'agit.

$16 / 01 / 2014$

Côté musique, mes activités de choriste continuent mais pour la viole, je passe une période creuse, car la personne qui nous avait rassemblé dans un ensemble baroque est rentrée en France, je n'ai plus donc la motivation de l'année dernière et je touche moins mon instrument.

Pour ces échanges, ton enthousiasme, ta curiosité, ta sensibilité, tes conseils, ton écoute Merci Aksel MERCI 


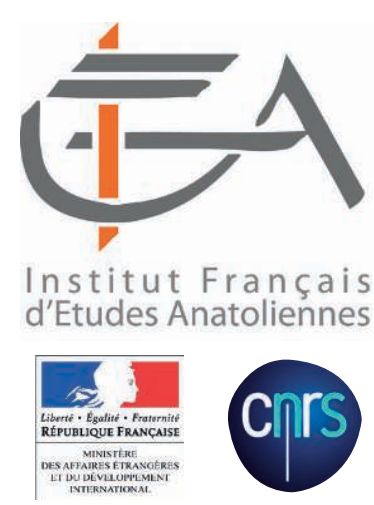

Figurine d'homme en terre cuite (Louvre, Tarse 314) (C) 2016 Réunion des musées nationaux / Tony Querrec. 